\title{
Primary Care Access During the COVID-19 Pandemic: a Simulated Patient Study
}

\author{
Michael Anne Kyle, MSN MPH RN $N^{1,2}$, Renuka Tipirneni, MD MSc ${ }^{3,4}$, Nitya Thakore, BA ${ }^{5}$, \\ Sneha Dave, $B A^{6}$, and Ishani Ganguli, MD MPH $H^{5,7}$
}

${ }^{1}$ Wyss House, Harvard Business School, Boston, MA, USA; ${ }^{2}$ Harvard Interfaculty Initiative in Health Policy, Boston, MA, USA; ${ }^{3}$ Division of General Medicine, Department of Internal Medicine, University of Michigan, Ann Arbor, MI, USA; ${ }^{4}$ Institute for Healthcare Policy and Innovation, University of Michigan, Ann Arbor, MI, USA; ${ }^{5}$ Brigham and Women's Hospital Division of General Internal Medicine and Primary Care, Boston, MA, USA; ${ }^{6} \mathrm{Health}$ Advocacy Summit, Indianapolis, IN, USA; ${ }^{7}$ Harvard Medical School, Boston, MA, USA.

\begin{abstract}
BACKGROUND: Primary care practices have experienced major strains during the COVID-19 pandemic, such that patients newly seeking care may face potential barriers to timely visits.

OBJECTIVE: To quantify availability and wait times for new patient appointments in primary care and to describe how primary care practices are guiding patients with suspected COVID-19.

DESIGN: Trained callers conducted simulated patient calls to 800 randomly sampled primary care practices between September 14, 2020, and September 28, 2020.

PARTICIPANTS: We extracted complete primary care physician listings from large commercial insurance networks in four geographically dispersed states between September 10 and 14, $2020(n=11,521)$. After excluding non-physician providers and removing duplicate phone numbers, we identified 2705 unique primary care physician practices from which we randomly sampled 200 practices in each region.
\end{abstract}

MAIN MEASURES: Primary care appointment availability, median wait time in days, and practice guidance to patients suspecting COVID-19 infection.

KEY RESULTS: Among 56\% of listed practices that had accurate contact information listed in the directory, 84\% offered a new patient in-person or virtual appointment. Median wait time was 10 days (IQR 3-26 days). The most common guidance in case of suspected COVID-19 was clinician consultation, which was offered in $41 \%$ of completed calls. Callers were otherwise directed to on-site testing (14\%), off-site testing (24\%), a COVID-19 hotline $(8 \%)$, or an urgent care/emergency department $(12 \%)$, while $2 \%$ of practices had no guidance to offer.

CONCLUSIONS: Despite resource constraints, most reachable primary care practices offered timely new patient appointments as well as direct COVID-19 care. Pandemic mitigation strategies should account for and support the central role of primary care practices in the community-based pandemic response.

KEY WORDS: COVID-19; ambulatory care; policy/economics; access; telemedicine

Received November 9, 2020

Accepted April 3, 2021

Published online April 26, 2021
J Gen Intern Med 36(12):3766-71

DOI: $10.1007 / \mathrm{s} 11606-021-06804-7$

(c) Society of General Internal Medicine 2021

\section{INTRODUCTION}

The COVID-19 pandemic has tested the capacity of US primary care practices, which were already experiencing resource constraints and declines in visit rates, ${ }^{1-4}$ with important implications for patient access. Primary care office visit volume plummeted in the first months of the pandemic. ${ }^{5}$ Temporary closures and declines in visit volume have destabilized the finances of primary care practices and caused clinicians and staff to be furloughed, laid off, or to leave or retire early, potentially creating immediate and long-term access challenges. ${ }^{6-9}$ Many practices have also had to adapt rapidly to offering virtual care in order to meet social distancing requirements and conserve limited personal protective equipment, which has dramatically changed the way both routine and urgent care are delivered. ${ }^{10}$

These strains on primary care are concerning given the importance of timely access during the pandemic to address patients' general and COVID-19-specific health needs. ${ }^{11}$ The growing share of US residents who did not have established primary care before the pandemic may now be seeking primary care for physical and mental health concerns that the pandemic may have magnified. ${ }^{12,13}$ Primary care clinicians routinely care for respiratory illnesses and may specifically serve as a first point of contact for many people infected with COVID-19, especially as they diagnose and support the majority whose COVID-19 infections are managed at home. ${ }^{11}$ Primary care also has a key role in public health, through primary prevention via influenza and now COVID-19 vaccination.

Timely access to care has been a longstanding challenge in primary care. Evidence suggests wait times for primary care visits were increasing prior to the pandemic, ${ }^{14}$ with a declining share of patients able to access a new patient appointment within a week, ${ }^{15}$ though new appointment wait times for privately insured patients did not significantly change after 
the Affordable Care Act brought an influx of newly insured patients. $^{16}$

With greater threats to and likely greater demand for primary care, it is unclear how potential new patients may experience establishing primary care during the COVID-19 pandemic.

Simulated patient studies provide a way to observe access to care from the patient's perspective, using direct observation to complement survey and administrative data assessments of access. ${ }^{17-21}$ We performed a simulated patient study 6 months into the COVID-19 pandemic to understand how potential new patients experience access to primary care. We examined availability and wait times for new patient appointments, availability and modalities of virtual visits, and practice guidance for patients with suspected COVID-19 symptoms. ${ }^{22}$

\section{STUDY DATA AND METHODS}

\section{Study Design}

Trained research team members made simulated patient calls to primary care practices posing as new patients seeking either a routine new patient appointment or a problem-based visit to address a newly identified primary care sensitive chronic condition that would pose risks if left untreated (i.e., hypertension). This study was deemed not to be human subjects research by the Harvard University institutional review board.

\section{Sample}

We constructed our sampling frame to mimic a search by a prospective new patient. We extracted publicly listed networks from the largest commercial insurer in four states (Arizona [AZ)], Illinois [IL], Massachusetts [MA], and North Carolina [NC]) in September 2020. We selected these states because they had varied geographic locations, COVID-19 mitigation policies, and COVID-19 caseload trajectories. In our selection of regions, we also took into account pragmatic factors such as the content and technical formatting of provider network listings to ensure consistency and rigor in our simulated patient calls.

In our search criteria, we specified primary care physicians who were accepting new patients. We restricted our search to a 25-mile search radius around the populous areas of Phoenix, AZ, Chicago, IL, Boston, MA, and Raleigh/Greensboro, NC metropolitan areas to replicate a realistic patient query and to maximize the sample size. After assembling our raw data, which consisted of the universe of available in-network primary care physicians for our simulated patients, we removed non-primary care physicians (specialist physicians and nurse practitioners or physician assistants), as non-physician providers are typically affiliated with a physician and we were interested in identifying unique primary care practices. We also removed duplicate phone numbers to avoid sampling the same practice multiple times. From each of the four networks, we randomly sampled 200 primary care practices for an overall sample of 800 .

\section{Measures}

We collected data on provider network accuracy (i.e., how often the listed phone number reached the intended primary care practice), appointment availability, appointment wait times, and availability and modalities of virtual visits. Finally, we inquired about practice guidance if patients suspected they had a COVID-19 infection.

\section{Data Collection}

Three trained callers placed simulated patient calls during regular business hours between September 14, 2020, and September 28, 2020. This period coincided with the 6-month mark of the COVID-19 pandemic in the USA. The callers used a standardized script with two scenarios: in the first, the caller posed as a new patient seeking a general new patient visit; in the second, the caller posed as a new patient with selfdiagnosed hypertension based on a recent blood pressure reading of 160/90. See Appendix for call script.

For each practice, we documented whether the initial call reached the primary care practice with the contact information provided by the listing. We classified practices as unreachable if they were out of scope (i.e., a medical site but not a primary care practice), if the listed phone number was disconnected, or if the listed phone number was a wrong number. If callers reached a messaging service or voice mailbox, they placed a second, follow-up call at a different time or date to that practice to ascertain appointment availability. If that second call also led to a messaging service or voice mailbox, the practice was also classified as unreachable.

For practices that were successfully reached, we documented whether an appointment was offered and the earliest available date. We documented if the earliest available appointment was with the listed physician or another provider. If an appointment was not offered, we documented the reason, e.g., practice not accepting new patients. We then asked whether the first or any appointment could be done virtually, and about the modalities of virtual visits offered (telephone, video, or both). Finally, we asked about practice guidance for patients suspecting a COVID-19 infection ("Can you tell me what the process is if I think I have Covid?").

\section{Analysis}

Primary outcomes were primary care appointment availability, wait times (days), availability of virtual visits, and practice guidance for patients suspecting a COVID19 infection. We examined the accuracy of patient-facing network listings by determining the percentage of practices that were not reachable and the associated reasons. 
For all primary outcomes, we used reachable practices as our denominator. We defined appointment availability as the offer of an appointment date. We defined wait times as the difference in calendar days between the call date and earliest available appointment date. We classified availability of virtual visits as follows: phone, video, both, not offered. We also asked whether or not first appointments could be conducted virtually. Finally, we timed the duration of each call, which included time spent on hold.

For the question about practices' COVID-19 guidance, we grouped the open-ended responses into six categories based on iterative coding by four members of the research team. We developed an initial set of categories through open coding and met as a team to discuss and apply codes together until we achieved concordance among all team members. Coding was subsequently applied to individual call responses and iteratively reviewed. We classified the following categories of COVID-19 guidance: [1] offered clinician consultation, i.e., with a physician or nurse; [2] offered on-site testing at the practice arranged by administrative staff; [3] referred to testing at another location, such as another location in the same health system, a commercial lab, or a pharmacy; [4] referred to a dedicated COVID-19 hotline for further assistance; [5] referred to urgent care or emergency department; [6] practice had no recommendations for what to do in case of suspected COVID19. Finally, we created a separate variable capturing whether COVID-19 testing was offered at the practice, since practices in any of these categories may have offered on-site setting.

In addition to reporting primary and secondary outcomes overall, we looked at outcomes by region and by condition scenario.

\section{RESULTS}

\section{Sample}

We extracted 11,521 complete primary care physician listings. After excluding non-physician providers and removing duplicate phone numbers, we identified 2705 unique primary care physician offices from which we randomly sampled 200 practices in each region (Fig. 1).

\section{Accuracy of Primary Care Practice Listings}

Of the 800 practices sampled, $447(56 \%)$ primary care offices were confirmed as reachable using the contact information listed in the directory (Fig. 1). The remaining $353(44 \%)$ practices were deemed unreachable. Of these 353 practices, $37 \%$ were out of scope, $10 \%$ had wrong numbers listed, $17 \%$ had disconnected phone lines listed, and $36 \%$ had numbers that went to messaging services or voice mailboxes on two occasions.

\section{Appointment Availability, Wait Times, and COVID-19 Guidance}

Among the 447 reachable practices, $84 \%$ offered a new patient appointment. The median wait time was 10 days (IQR 3-26 days). Of the 376 new patient appointments offered, $68 \%$ were with the listed provider. When appointments were not offered, reasons included the following: practice not accepting new patients (8\%); practice did not accept insurance (3\%); and other $(5 \%)$. Mean call duration including time on hold was 5 min (range 0.3-21 min).

Of primary care practices reached, $75 \%$ offered virtual visits including $25 \%$ which offered virtual visits for the initial visit. Of the $75 \%$ offering virtual visits, $36 \%$ offered telephone or video, $28 \%$ offered video alone, and $10 \%$ offered telephone visits alone (numbers do not exactly add up to $75 \%$ due to rounding).

The most common guidance in the case of suspected COVID-19 was clinician consultation, which was offered in $41 \%$ of completed calls for which this information was available (383 of 447 reachable practices) (Table 1). Callers were otherwise directed to on-site testing (14\%), referred to off-site testing (24\%), offered a COVID-19 hotline (8\%), or sent to urgent care/emergency department (12\%). Only $2 \%$ of practices had no guidance for what to do in the case of suspected COVID-19. About one fifth (22\%) of practices overall said they offered COVID-19 testing on-site.

\section{Variations by Region}

Accuracy of network listings varied by region. Of the 200 practices sampled in each state, $55 \%$ of practices were reachable in Arizona (AZ), 39\% in Illinois (IL), 65\% in Massachusetts (MA), and $66 \%$ in North Carolina (NC).

Among reachable practices, appointments were offered by $88 \%$ of primary care practices in AZ, $92 \%$ in IL, $78 \%$ in MA, and $82 \%$ in NC. Median appointment wait time in MA was 24 days (IQR 5-48 days), more than twice as long as in any other state. Median wait times were similar in other states: 7 days (IQR 3-13 days) in AZ, 7 days (IQR 3-18 days) in IL, and 10 days (IQR 3-21 days) in NC.

Availability of virtual visits was slightly higher in AZ $(83 \%)$ but similar across the other states $(75 \%$ in IL, $73 \%$ in $\mathrm{MA}$, and $72 \%$ in NC). With respect to virtual visit modality, the option for either phone or video was most common in IL (48\%), MA (36\%), and $\mathrm{NC}(40 \%)$, whereas in $\mathrm{AZ}$ video alone was most common (38\%).

Clinician consultation was the most common guidance for suspected COVID-19 in all states, recommended as a next step by $33 \%$ of practices in AZ, $47 \%$ in IL, $40 \%$ in MA, and $44 \%$ in NC.

\section{Variations by Health Condition Scenario}

There were no notable differences in results by visit type (general new patient visit versus hypertension). Wait times 


\begin{tabular}{|c|c|c|}
\hline \multicolumn{3}{|c|}{$\begin{array}{l}\text { Practices called } \\
\qquad N=800\end{array}$} \\
\hline \multicolumn{2}{|l|}{$\begin{array}{l}\text { Reachable practices } \\
\qquad \mathrm{N}=447\end{array}$} & $\begin{array}{l}\text { Unreachable practices } \\
\qquad \mathrm{N}=353\end{array}$ \\
\hline $\begin{array}{l}376(84 \%) \text { appointment offered } \\
10 \text { day (IQR 3-26) median wait time for first available appointment } \\
255(57 \%) \text { appointment offered with listed physician } \\
337(75 \%) \text { virtual visits available } \\
44(10 \%) \text { phone visits only } \\
125(28 \%) \text { video visits only } \\
163(36 \%) \text { phone and video visits } \\
111(25 \%) \text { virtual first visit available }\end{array}$ & $\begin{array}{l}71(16 \%) \text { appointment not offered } \\
37(8 \%) \text { not accepting new patients } \\
12(3 \%) \text { not accepting insurance } \\
2(0 \%) \text { urgent care/walk-in } \\
22(5 \%) \text { other }\end{array}$ & $\begin{array}{l}129(37 \%) \text { out of scope } \\
34(10 \%) \text { wrong number } \\
60(17 \%) \text { disconnected } \\
128(36 \%) \text { messaging service }\end{array}$ \\
\hline
\end{tabular}

Figure 1 Simulated patient study results showing network accuracy and new patient visit availability, wait times, and availability.

for both conditions were similar: 10 days (IQR 3-27 days) for general new patient visit requests and 9 days (IQR 4-24 days) for new patients requesting a visit based on an elevated blood pressure reading.

\section{DISCUSSION}

In this simulated patient study of new primary care patient access during the COVID-19 pandemic, we found that despite high inaccuracy rates in patient-facing provider directories, timely primary care visits were largely available among reachable practices. In addition to routine services, most primary care practices provided direct COVID-19 consultation and/or on-site testing.

While much attention during the pandemic has been focused on inpatient and critical care resources, our results demonstrate the central and active role primary care practices are playing in the COVID-19 response. Of the reachable practices in our sample, more than half offered patients the chance to speak with a clinician or come to the practice site for testing as an immediate next step for patients suspecting a COVID-19 infection. Otherwise, patients were referred to other testing or treatment resources, such as off-site testing, or - most common in large health systems - a centralized hotline. Only $12 \%$ referred patients to higher acuity care such as urgent care or emergency departments, while $2 \%$ of prac-

Table 1 Practice Guidance in Cases of Suspected COVID-19

\begin{tabular}{lc}
\hline \hline & $N=\mathbf{3 8 3} * \mathbf{( \% )}$ \\
\hline Next step advised & \\
Clinician consultation & $156(41 \%)$ \\
On-site testing & $53(14 \%)$ \\
Referred to off-site testing & $92(24 \%)$ \\
COVID-19 hotline & $30(8 \%)$ \\
Urgent care/ED/hospital & $45(12 \%)$ \\
No recommendation & $7(2 \%)$ \\
Practice offers on-site testing ${ }^{\dagger}$ & $85(22 \%)$ \\
\hline Notes: *Includes all practices for which data available. For 65 \\
practices, the call was terminated before the question could be asked. \\
Hncludes practices that advised next steps other than on-site testing. $_{\text {This was based on conservation definition in which practices were }}$ \\
credited if they explicitly mentioned this option
\end{tabular}

tices offered no guidance. These results demonstrate that despite resource constraints, primary care practices are leading outpatient and community-based management of COVID-19 infection, which can help keep patients out of overwhelmed emergency departments and hospitals. ${ }^{9,}{ }^{23}$ Policymakers and health care leaders should better account for and support primary care's critical role in pandemic mitigation strategies. ${ }^{9}$, 23, 24 Primary care practices' finances remain vulnerable despite enhanced telehealth reimbursement and pandemic relief funds, and additional financial relief may be needed to support their ongoing operations. ${ }^{7}$

We found that despite strains on primary care practices, most practices offered relatively timely new patient primary care visits 6 months into the pandemic, with a median wait time of 10 days. Wait times were overall comparable to prior studies of wait times: slightly longer than those reported in two large primary care simulated patient studies conducted after Affordable Care Act coverage expansions that increased demand for care, ${ }^{20,16}$ and slightly shorter than another national wait times study with smaller sample sizes. ${ }^{14}$ Regional variation in wait times was similar to that seen in prior studies, with Massachusetts having longer primary care wait times compared to other states and regions. Because the pandemic has affected both demand for and availability of primary care, our wait time findings may reflect a number of factors. Evidence on changes in patient demand is mixed: while primary care visits seemed to rebound above pre-pandemic baseline after a decline in Spring $2020^{5}$, there is also evidence that patients delayed or avoided medical care because of the pandemic. ${ }^{25}$ Regarding availability, it is possible our wait times reflect secular declines in primary care workforce compared to earlier studies, or declines in capacity precipitated by pandemicspecific constraints. ${ }^{23,} 26$

Notably, virtual visits played a role in preserving access to primary care, including nearly all clinician consultations for suspected COVID-19 - suggesting a need for maintaining payer and regulatory support for virtual care. The Centers for Medicare and Medicaid Services (CMS) offered temporary payment parity across in-person, telephone, and video visits in March 2020, and many private insurers followed suit. 
However, telehealth payment and policies were made under the aegis of the public health emergency declaration and permanent legislation will be needed to support virtual access to care long-term. ${ }^{27}$

Consistent with prior research, ${ }^{17,} 28,29$ we found patientfacing provider listings were rife with inaccuracies, even though we collected provider network data within a week of conducting calls. This suggests insurers and practices should take greater responsibility for the information provided to patients, since inaccurate listings place undue stress and time burden on all prospective patients and especially those for whom navigating health care is already a challenge. In recent guidance, CMS indicated they plan to decrease Medicare Advantage plan star ratings if provider directories are inaccurate.

Looking forward, policymakers should recognize the role primary care has played in pandemic response and generously fund primary care capacity building. The early rollout of the COVID-19 vaccine has been rocky, and notable for the lack of attention to primary care as a channel for outreach and vaccination. Our study shows primary care practices were largely responsive to new patients and had made significant COVID-19specific adaptations despite difficult conditions - indications that primary care practices are poised to make major contributions to a successful vaccination campaign given the appropriate policy and payment support.

\section{Limitations}

Our findings are limited to appointment availability for patients with commercial insurance. We chose commercial networks to capture the "best case" scenario for access; patients with other types of insurance, such as Medicaid, might face more limited access. However, we note evidence of primary care access barriers even among commercial populations. ${ }^{3,4}$ While our study sample is geographically dispersed and includes major metropolitan centers across the country, it does not represent a national random sample across insurance or network types, nor does it capture rural populations. We collected data over 14 days to ensure comparability of data within our sample, but this cross-sectional analysis has limitations: COVID19 prevalence, virtual visit policies, and other environmental conditions continue to evolve. Furthermore, we cannot isolate the relationship between regional transmission and visit availability and/or telemedicine prevalence; differences between regions would be affected by factors such as baseline availability, sequelae of pent-up or reduced demand based on the earlier course of the pandemic, and state telehealth payment/policy. Because the pandemic was an unforeseen shock, we were unable to collect pre-period data, but we can use these results to benchmark changes in access going forward. Finally, we did not systematically collect data that would allow us to compare wait times between in-person and virtual visits.
Study strengths include a large geographically diverse sample collected through direct extraction of publicly listed networks, a robust simulated patient call protocol developed and refined to capture timely data on primary care access during the pandemic, a negligible rate of incomplete or unclassifiable calls which have been reported as high as $10 \%$ in other studies, ${ }^{30-32}$ and qualitative information on the COVID-19 response being conducted by primary care practices.

\section{CONCLUSION}

Despite limited accuracy of patient-facing provider listings, timely new patient appointments were typically available when simulated patients were able to reach a primary care practice. Primary care practices are playing an active role in COVID-19 testing and management, which should be accounted for in pandemic mitigation strategies.

Corresponding Author: Michael Anne Kyle, MSN MPH RN; Wyss House, Harvard Business School, Boston, MA, USA (e-mail: mkyle@hbs.edu).

Supplementary Information The online version contains supplementary material available at https://doi.org/10.1007/s11606-02106804-7.

\section{Declarations:}

Conflict of Interest: The authors declare no conflicts of interest. Kyle is supported by a Harvard Business School Doctoral Fellowship. Dr. Tipirneni is supported by a K08 Clinical Scientist Development Award from the National Institute on Aging (K08AG056591). Dr. Ganguli reports consulting fees from Haven, Blue Cross Blue Shield Massachusetts, and F-Prime Capital as well as a grant from International Business Machines (IBM) Corporation, all of which are unrelated to this work

\section{REFERENCES}

1. Bodenheimer T, Pham HH. Primary Care: Current Problems And Proposed Solutions. Health Aff (Millwood). 2010;29(5):799-805. https:// doi.org/10.1377/hlthaff.2010.0026

2. Ganguli I, Lee TH, Mehrotra A. Evidence and Implications Behind a National Decline in Primary Care Visits. J Gen Intern Med. Published online June 26, 2019. https://doi.org/10.1007/s11606-019-05104-5

3. Ganguli I, Shi Z, Orav EJ, Rao A, Ray KN, Mehrotra A. Declining Use of Primary Care Among Commercially Insured Adults in the United States, 2008-2016. Ann Intern Med. 2020;172(4):240-247. https://doi.org/10. 7326/M19-1834

4. Rao A, Shi Z, Ray KN, Mehrotra A, Ganguli I. National Trends in Primary Care Visit Use and Practice Capabilities, 2008-2015. Ann Fam Med. 2019;17(6):538-544. https://doi.org/10.1370/afm.2474

5. Mehrotra A, Chernew M, Linetsky D, Hatch H, Cutler D, Schneider EC. The Impact of the COVID-19 Pandemic on Outpatient Care: Visits Return to Prepandemic Levels, but Not for All Providers and Patients. https://doi.org/10.26099/41xy-9m57.

6. What Impact Has COVID-19 Had on Outpatient Visits? https://doi.org/ 10.26099/ds9e-jm36.

7. Basu S, Phillips RS, Phillips R, Peterson LE, Landon BE. Primary Care Practice Finances In The United States Amid The COVID-19 Pandemic. 
Health Aff (Millwood). Published online June 25, 2020. https://doi.org/ 10.1377/hlthaff.2020.00794.

8. How Small Physician Practices are Struggling to Survive During Coronavirus Pandemic - The New York Times. Accessed 21 July 2020. https:// www.nytimes.com/2020/05/05/health/coronavirus-primary-care-doctor.html.

9. COVID Survey. The Larry A. Green Center. Accessed 19 October 2020. https://www.green-center.org/covid-survey.

10. How The Rapid Shift To Telehealth Leaves Many Community Health Centers Behind During The COVID-19 Pandemic | Health Affairs. Accessed 21 July 2020. https://www.healthaffairs.org/do/10.1377/ hblog20200529.449762/full/.

11. World Health Organization Regional Office for the Western. Role of primary care in the COVID-19 response. Published online April 21 2020. Accessed 13 October 2020. https://apps.who.int/iris/handle/ 10665/331921

12. Levine DM, Landon BE, Linder JA. Quality and Experience of Outpatient Care in the United States for Adults With or Without Primary Care. JAMA Intern Med. 2019;179(3):363. https://doi.org/10.1001/ jamainternmed.2018.6716

13. Czeisler MÉ. Mental Health, Substance Use, and Suicidal Ideation During the COVID-19 Pandemic - United States, June 24-30, 2020. MMWR Morb Mortal Wkly Rep. 2020;69. https://doi.org/10.15585/mmwr. mm6932a

14. 2017 Survey of Physician Appointment Wait Times. Merritt Hawkins. 36 https://www.merritthawkins.com/uploadedFiles/MerrittHawkins/Pdf/ mha2017waittimesurveyPDF.pdf.

15. Polsky D, Candon M, Saloner B, et al. Changes in Primary Care Access Between 2012 and 2016 for New Patients With Medicaid and Private Coverage. JAMA Intern Med. 2017;177(4):588. https://doi.org/10.1001/ jamainternmed.2016.9662

16. Rhodes KV, Basseyn S, Friedman AB, Kenney GM, Wissoker D, Polsky D. Access to Primary Care Appointments Following 2014 Insurance Expansions. Ann Fam Med. 2017;15(2):107-112. https://doi.org/10. 1370/afm.2043

17. Haeder SF, Weimer DL, Mukamel DB. Secret Shoppers Find Access To Providers And Network Accuracy Lacking For Those In Marketplace And Commercial Plans. Health Aff (Millwood). 2016;35(7):1160-1166. https:// doi.org/10.1377/hlthaff.2015.1554

18. Saloner B, Polsky D, Kenney GM, Hempstead K, Rhodes KV. Most Uninsured Adults Could Schedule Primary Care Appointments Before The ACA, But Average Price Was \$160. Health Aff (Millwood). 2015;34(5):773-780. https://doi.org/10.1377/hlthaff.2014.1258

19. Tipirneni R, Rhodes KV, Hayward RA, Lichtenstein RL, Reamer EN, Davis MM. Primary Care Appointment Availability For New Medicaid Patients Increased After Medicaid Expansion In Michigan. Health Aff (Millwood). 2015;34(8):1399-1406. https://doi.org/10.1377/hlthaff 2014.1425
20. Polsky D, Richards M, Basseyn S, et al. Appointment Availability after Increases in Medicaid Payments for Primary Care. N Engl J Med. 2015;372(6):537-545. https://doi.org/10.1056/NEJMsa1413299

21. Rhodes KV, Kenney GM, Friedman AB, et al. Primary care access for new patients on the eve of health care reform. JAMA Intern Med. 2014;174(6):861-869. https://doi.org/10.1001/jamainternmed.2014.20

22. After COVID-19: How To Rejuvenate Primary Care For The Future । Health Affairs. Accessed 21 July 2020. https://www.healthaffairs.org/ do/10.1377/hblog20200515.372874/full/.

23. Ganguli I. Covid-19 is harshly testing our capacities as physicians and medical staffers. Washington Post. https://www.washingtonpost.com/ national/health-science/covid-19-is-harshly-testing-our-capacities-asphysicians-and-medical-staffers/2020/03/26/8f4bea42-6e11-11 eab148-e4ce3fbd85b5_story.html. Accessed 19 October 2020

24. A safer world starts with strong primary healthcare. The BMJ. Published October 26, 2020. Accessed 4 November 2020. https://blogs.bmj.com/ bmj/2020/10/26/a-safer-world-starts-with-strong-primary-healthcare/

25. Czeisler MÉ. Delay or Avoidance of Medical Care Because of COVID-19Related Concerns - United States, June 2020. MMWR Morb Mortal Wkly Rep. 2020;69. https://doi.org/10.15585/mmwr.mm6936a4

26. Machado SR, Jayawardana S, Mossialos E, Vaduganathan M. Physician Density by Specialty Type in Urban and Rural Counties in the US 2010 to 2017. JAMA Netw Open. 2021;4(1):e2033994. https://doi.org/ 10.1001/jamanetworkopen.2020.33994

27. Verma S. Early Impact Of CMS Expansion Of Medicare Telehealth During COVID-19 | Health Affairs. Health Affairs Blog. Accessed 28 December 2020. https://www.healthaffairs.org/do/10.1377/hblog20200715. 454789/full/

28. State Standards for Access to Care in Medicaid Managed Care (OEI-0211-00320; 09/14). 34

29. Access to Care: Provider Availability in Medicaid Managed Care (OEI-O213-00670; 12/14). 31.

30. Asplin BR. Insurance Status and Access to Urgent Ambulatory Care Follow-up Appointments. JAMA. 2005;294(10):1248. https://doi.org/10 $1001 /$ jama.294.10.1248

31. Blanchard J, Ogle K, Thomas O, Lung D, Asplin B, Lurie N. Access to Appointments Based on Insurance Status in Washington, D.C. J Health Care Poor Underserved. 2008;19(3):687-696. https://doi.org/10.1353/ hpu.0.0036

32. Access of Medicaid Recipients to Outpatient Care. N Engl J Med 1994:330(20):1426-1430. https://doi.org/10.1056/ NEJM199405193302007

Publisher's Note: Springer Nature remains neutral with regard to jurisdictional claims in published maps and institutional affiliations. 